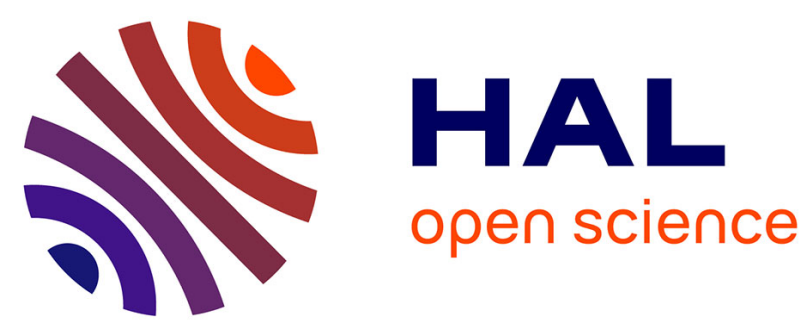

\title{
Vagus nerve stimulation for treatment-resistant depression: is this therapy distinct from other antidepressant treatments?
}

Andrei Vlaicu, Mihaela Bustuchina Vlaicu

\section{- To cite this version:}

Andrei Vlaicu, Mihaela Bustuchina Vlaicu. Vagus nerve stimulation for treatment-resistant depression: is this therapy distinct from other antidepressant treatments?. International Journal of Psychiatry in Clinical Practice, 2020, 24 (4), pp.349 - 356. 10.1080/13651501.2020.1779751 . hal-03009393

\section{HAL Id: hal-03009393 \\ https://hal.sorbonne-universite.fr/hal-03009393}

Submitted on 24 Nov 2020

HAL is a multi-disciplinary open access archive for the deposit and dissemination of scientific research documents, whether they are published or not. The documents may come from teaching and research institutions in France or abroad, or from public or private research centers.
L'archive ouverte pluridisciplinaire HAL, est destinée au dépôt et à la diffusion de documents scientifiques de niveau recherche, publiés ou non, émanant des établissements d'enseignement et de recherche français ou étrangers, des laboratoires publics ou privés. 


\section{Vagus nerve stimulation for treatment-resistant depression: is this therapy distinct from other antidepressant treatments?}

Andrei Vlaicu \& Mihaela Bustuchina Vlaicu 


\title{
Vagus nerve stimulation for treatment-resistant depression: is this therapy distinct from other antidepressant treatments?
}

\author{
Andrei Vlaicu ${ }^{\mathrm{a}}$ and Mihaela Bustuchina Vlaicu ${ }^{\mathrm{b}, \mathrm{c}}$ \\ ${ }^{a}$ Service of Psychiatry, Hospital Andre Breton, Saint-Dizier, France; ${ }^{b}$ Neurosurgery Department, Hospital Pitié Salpêtrière, Paris, France; ${ }^{c}$ INSERM, \\ U955, The Translational Psychiatry Laboratory, Créteil, France
}

\begin{abstract}
Background: The treatment-resistant depression (TRD) is a very disabling disease.

Objective: The aim of this article is to provide an overview of the therapeutic activity of vagus nerve stimulation (VNS) therapy system in TRD. We summarised the progress made during the last decade in this area.

Methods: We conducted a non-systematic review on the efficacy and safety of the VNS therapy for this disease. We analysed the results from acute and long-term studies that utilised this technique. Major electronic databases were searched.

Results: The patients with TRD may show acute and long-term benefit when treated with this technique. There are promising results for VNS therapy for these patients. The level of evidence as an acute treatment option is only 3 , but as chronic treatment is 2 . This therapy should be offered as an added longterm treatment option for patients with chronic and recurrent difficult to treat depression.

Conclusions: The antidepressant effects of this procedure remain controversial. The clinical trials have produced mixed results, but VNS therapy for TRD has two distinct features that differentiate it from other antidepressant treatments: a sustained therapeutic response obtained in highly resistant depressive disorders, a favourable safety profile and guaranteed compliance.
\end{abstract}

\section{ARTICLE HISTORY}

Received 26 September 2019

Revised 7 January 2020

Accepted 1 June 2020

\section{KEYWORDS}

Neurostimulation; treatment-refractory depression; vagus nerve stimulation; neuroprotection; technological advances; clinical trials

\section{Introduction}

Major depression is now recognised as a highly prevalent, chronic, recurrent disorder with high rates of morbidity and mortality. Currently available therapeutic interventions for treatment-resistant depression (TRD), including high-dose antidepressants, switch, combination, augmentation strategies, psychotherapy, and electroconvulsive therapy (ECT) are less than ideal. In this context, for many of these patients, neuromodulation therapy with vagus nerve stimulation (VNS) therapy could be a real alternative.

The first description in the literature about use of VNS therapy in humans was made in 1990 (for the treatment of drug-resistant epilepsy) (Penry and Dean 1990). Since June 1994, the VNS therapy system has been approved as adjuvant therapy for drugresistant epilepsy in all EU member countries. VNS was approved in the United States by the Food and Drug Administration (FDA) for treatment of resistant epilepsy in 1997.

Mood improvements observed in patients treated with VNS for epilepsy suggested that this therapy might also be beneficial in depression. In March 2001, Cyberonics was granted the agreement to begin commercial distribution of the VNS therapy system for the treatment of depression in members of the European Community (EC), and one month later for Canada. VNS was approved by the FDA in the United States in 2005 for the adjunct long-term treatment of chronic or recurrent depression for adult patients experiencing a major depressive episode who had failed to respond to four or more adequate antidepressant treatments (Table 1).

\section{The mechanism of the vagus nerve stimulation therapy system ${ }^{\mathrm{TM}}$}

The vagus nerve is a cranial mixed nerve composed of $20 \%$ efferent fibres and $80 \%$ afferent fibres, that transmit nerve impulses from the periphery to the brain. As in epilepsy, the exact mechanisms of action of VNS are not fully understood. Putative antiseizure mechanisms are mediated by altered vagal afferent activities, and probably include altered activities in the reticular activating system, the central autonomic network, the limbic system, and the diffuse noradrenergic projection system. Studies have shown that activation of the vagus nerve, particularly the afferent fibres, influences the brain areas involved in depression. Electrical stimulation of the vagus nerve centrally stimulates the nucleus tractus solitarius (NTS), which sends fibres to connect directly or indirectly to different regions of the brain.These regions include the dorsal raphe nucleus (DRN), the locus coeruleus (LC), the amygdala, the hippocampus, the thalamus and the orbitofrontal cortex. The NTS can modulate multiple regions of the brain via its neuronal connections to anatomically distributed subcortical and cortical regions of the brain that are involved in mood regulation. The stimulation of the NTS beam, which itself projects on LC (noradrenergic) and on NR (serotoninergic), results in changes in prefrontal cortical activity. Norepinephrine (NE) and serotonin (5-HT) levels of neurons in the LC and of dorsal nucleus of the raphe (NR) increased with treatment with VNS (Ruffoli et al. 2011). According to several authors, the modulation of the neuronal pathways associated with the regulation of the mood by the NTS seems to constitute an attractive mechanism of action (Nemeroff et al. 2006). 
Table 1. History of VNS therapy ${ }^{\circledR}$.

\begin{tabular}{ll}
\hline 1988 & First patient implanted (epilepsy) \\
$1988-2005$ & E-01-E-05 (epilepsy studies; 439 patients) \\
1994 & CE-Mark approval epilepsy \\
1997 & FDA approval epilepsy \\
2000 & Pilot study depression (early observation mood improvement) \\
$2000-2009$ & D-01, D-02 and D-03 (depression studies) \\
2001 & CE-Mark Approval Depression (Europe and Canada) \\
2005 & FDA approval depression \\
\hline
\end{tabular}

Changed activity in the orbital and ventromedial prefrontal cortices has also been recorded (Chae et al. 2003; Müller et al. 2013). In its mode of action, VNS modulates or fundamentally alters the concentrations of neurotransmitters (especially 5-HT, NE, GABA and glutamate) and their metabolites while producing changes in the functional activity of CNS regions (Hulsey et al. 2019). The results of human studies of CSF metabolites seem interesting: the metabolites of $\mathrm{NE}, 5 \mathrm{HT}$ and dopamine (DA) were evaluated before and after 24 weeks of stimulation by the VNS. Only the increase of homovanillic acid (HVA), a metabolite of DA has been observed, suggesting a possible dopaminergic mechanism of VNS (Grimonprez et al. 2015).

Neuroimaging studies have shown evidence that activity in the thalamus and cortex in depressed patients is altered by VNS therapy (Nahas et al. 2007). VNS can induce synchronisation of orbitofrontal activity on the EEG and it can induce emergence of frontal slow waves (Groves and Brown 2005).

The location of vagus nerve activity in the brain has been observed through studies of regional cerebral metabolism with positron emission tomography (Conway et al. 2013). Brain imaging studies have demonstrated metabolic changes in the prefrontal cortex and in limbic structures relevant to mood regulation, possibly through the modulation of monoaminergic neurotransmission. Probably that VNS would exert its antidepressant effects through a rapid increase in monoamine concentration, which then could improve neuronal plasticity in the hippocampus (Liu et al. 2017). The acute and chronic stimulation can induce persistent changes in neurons at this level (Revesz et al. 2008). It will also be possible to restore corticolimbic networks, which are disturbed in depressed patients. The formation of new synapses could strengthen these networks. In current treatment of TRD models, dysregulation of several interconnected structures in the frontal and limbic circuit has been hypothesised (in which the hippocampus plays a major role) (Perini et al. 2017). There is a hypothesis of the desynchronisation of neuronal activity by the modulation of neurotransmitter release, hippocampal plasticity and anti-inflammation action (Aalbers et al. 2011). In the hippocampal plasticity hypothesis of depression, chronic stress leads to atrophy and synaptic changes in limbic brain. VNS increases the expression of neurotrophic and growth factors brain-derived neurotrophic factor (BDNF) (Furmaga et al. 2012) and basic fibroblast growth factor (bFGF), (Follesa et al. 2007) which promote the neuroplasticity. Clinical studies have proven that therapeutic effects appear after several months of treatment and the plasticity hypothesis explains its mechanism. Recovery of networks that dysfunctioned in depressed subjects could provide an explanation for the therapeutic delay of VNS in the treatment of depression. In conclusion, the mechanism of action of VNS remains to be further elucidated (Vonck and Larsen 2018). The preclinical research reveals VNS induced effects on neurotransmitter expression, in the cerebral blood flow, desyncronisation changes EEG rhythms, on brain plasticity, the autonomic nervous system and the inflammatory response (Conway and Xiong 2018) (Figure 1).

\section{The device and the procedures for the implantation of the vagus nerve stimulation}

The VNS system comprises an implantable pulse generator (IPG), which is surgically inserted underneath the skin of the chest, connected to a helical bipolar cuff electrode (e.g., VNS therapy, Cyberonics) with a bipolar stimulation pattern, either with a fixed nonadaptive stimulation or on-demand adaptive stimulation triggered by the patient. The electrode is placed at the cervical level wrapped typically around the left cervical branch of vagus nerve. Intermittent electrical currents are sent from the generator to the vagus nerve and via the NTS to various regions of the brain. The electrodes are capable of delivering at low frequency pulses to the left vagus nerve. The pacemaker is typically activated for 30 seconds ON and five minutes OFF. Communication with the pulse generator occurs with the help of a tablet programmer and a wand. In depression, the recommended dosing parameters consist of a current intensity of $1.00-2.00 \mathrm{~mA}$, a pulse width of $250 \mu \mathrm{s}$ and a pulse frequency of $20 \mathrm{~Hz}$. These settings are based on use in clinical studies and empirical evidence. Optimal treatment parameters for VNS remain a research question. In an RCT of open-label VNS $(N=331)$ comparing low $(0.25 \mathrm{~mA}$ current, $130 \mathrm{~ms}$ pulse width), medium $(0.5-1.0 \mathrm{~mA}, 250 \mathrm{~ms})$ or high $(1.25-1.5 \mathrm{~mA}$, $250 \mathrm{~ms}$ ) electrical outputs, higher electrical charges were correlated with better improvement in depressive symptoms (Aaronson et al. 2013). More sustained antidepressant responses and less frequent suicide attempts were reported in the medium- and high stimulation groups than the low-dose group. We note that the effect is maintained and even increases with time (Cristancho et al. 2011). Implantation of the VNS Therapy system is similar to the implant of a pacemaker and it is performed under general anaesthesia. Left cervical implantation at the vagus nerve is believed to minimise potential cardiac effects, such as bradycardia or asystole (mainly mediated by the right vagus nerve). During the first months of treatment, optimisation of dosing parameters occurs, therefore, regular visits are required at the beginning of therapy. In the long-term, yearly check-ups are advised to ensure the functioning of the device and to adjust parameters if necessary. The stimulation parameters can be adjusted to avoid undesirable effects. Tolerance often improves with chronic stimulation. Once implanted, the VNS device can remain operational for a minimum of 7 years (Cyberonics 2015). The delivery of the treatment is automatic. The patient should receive safety information about the VNS, possible side-effects and risk. Occasionally removal of the VNS system may be necessary. Since VNS is adjuvant to antidepressant therapy, it is strongly advised to maintain the dosage of all antidepressant drugs at least for the first three months of stimulation, before attempting to reduce doses or switch medications. Once the VNS system is adjusted, depending on the patient's response and how it tolerates the implant, a schedule of followup visits will be established. At each patient visit, the pulse generator must be queried, using the appropriate version of the VNS system programming software. The treatment with the VNS system should not cause discomfort or unpleasant side effects. If the patient has an intolerable side effect, try routinely to reduce the output current $(\mathrm{mA})$. Additionally, it will be necessary to explain to patients and caregivers how to apply the magnet to turn off the pulse generator if an adverse reaction becomes intolerable.

\section{Contraindications}

A history of left or bilateral cervical vagotomy is a major contraindication. In patients stimulated with VNS, whole body MRI 


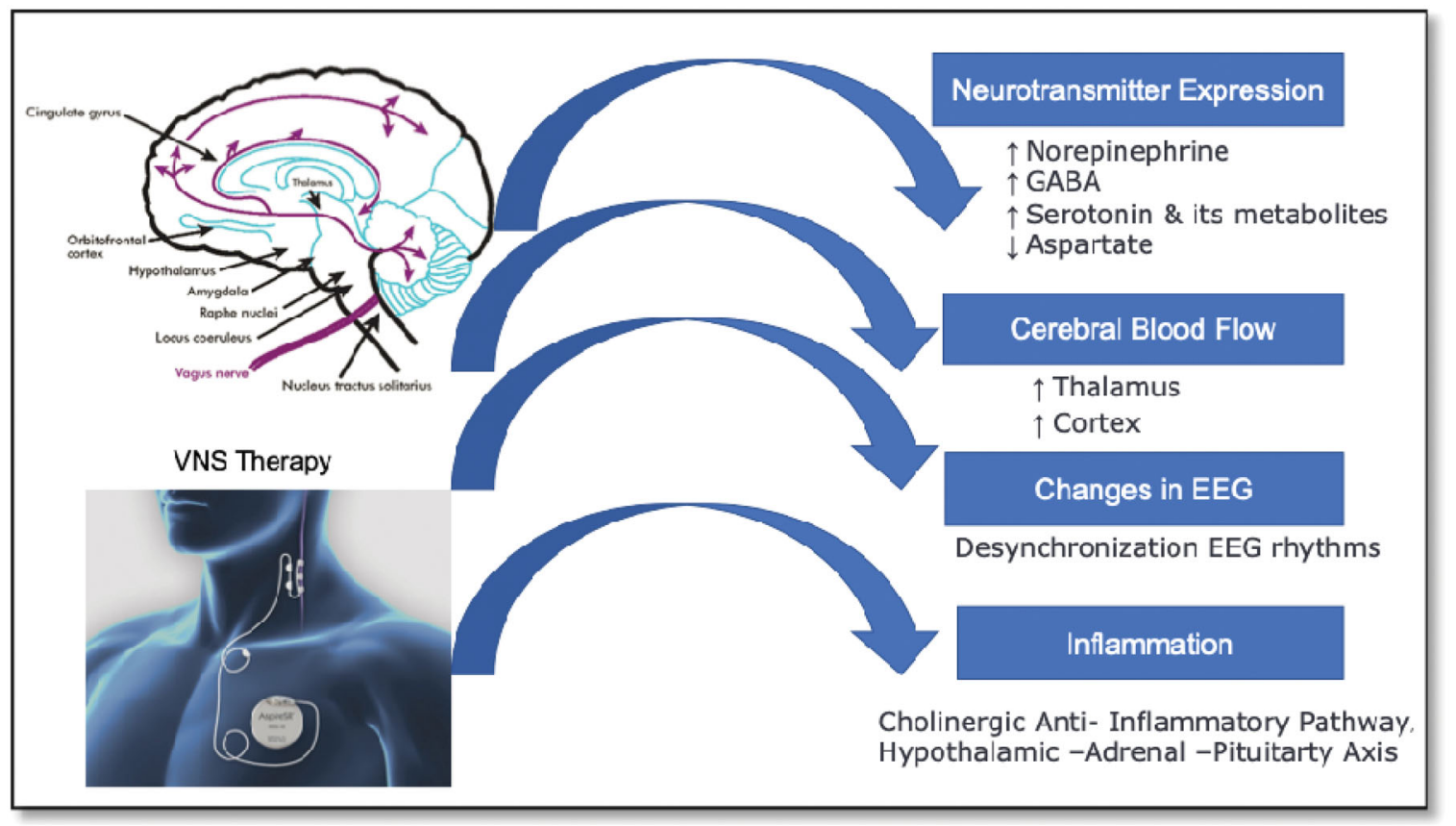

Figure 1. VNS therapy works via several pathways.

cannot be performed. The realisation of a cerebral MRI remains possible but requires specific equipment and procedures. Ultrasound therapies should not be used, but ultrasound is accepted. Metal detectors, microwave ovens, mobile phones and other electrical or electronic devices do not affect the VNS. The VNS system can affect the operation of other implanted devices such as pacemakers and defibrillators. This technique is compatible with the use of psychotropic drugs and with ECT.

\section{Safety and tolerance}

Surgical complications are rare but may include some infection risks as well as pain at the site of incision. Asystole during implantation is a very rare complication (1/1000 implants) but clearly represents a serious adverse event. However, no deaths were reported, and patients were able to safely use the VNS after the operation. Adverse events related to stimulation include: hoarseness of the voice (most common, but of low severity), feeling dyspnoea, paresthaesia, increased cough, laryngeal spasm and nausea/vomiting. In general, these manifestations appear only during stimulation, and they seem to be related to the intensity of the output current. Their effects diminish over time (Mehta et al. 2018).

\section{Patient selection}

Patient selection is an important step. First, the diagnosis of TRD should be confirmed: the course of the disease prior to the introduction of this device should clearly indicate that the long-term antidepressant treatment was well prescribed, that the subject was treated adequately with several treatments and has a significant disability due to his illness. This involves ensuring that the patient adheres to the treatment, that he has been given an adequate dose and of sufficient duration for each stage of the treatment, but that this has failed.

\section{What are the conclusions of the studies with vagus nerve stimulation?}

After studying the literature, it seems important to describe the studies that led to the development of VNS therapy for TRD (Rush and Siefert 2009). In the open pivotal feasibility study (D-01), of the 59 subjects with evaluable data, 31\% were responders at the end of the short-term study, 10 weeks after implantation (Rush et al. 2000). The response is the primary measure of effectiveness, which is defined as at least $50 \%$ improvement in the score on the HDRS-28 scale. Other measurements of depressive symptoms (CGI, MADRS, BDI, IDS-SR) and quality of life (MOS-36) corroborated the scores obtained on the HDRS28 scale. Sackheim et al. concluded that the mean response time to treatment was 48.1 days (Sackeim et al. 2001). Nahas et al. conducted a two-year outcome study for the same population (Nahas et al. 2005). The results indicate that remission rates were $15 \%$ at 3 months, $27 \%$ at 12 months and $22 \%$ after 2 years. Response rates were $31 \%, 44 \%$ and $42 \%$, respectively, at 3, 12 and 24 months. The authors concluded that treatment with VNS may show a long-term benefit. The clinical advantage over time is defined as extraordinary $(\geq 75 \%$ improvement for the HDRS-24 scale), very significant $(50 \%$ to $<75 \%$ ), significant $(25 \%$ to $<50 \%$ ), minimal ( $\%$ to $<25 \%$ ) or aggravated $(<0 \%)$. It is interesting to note that at 2 years the response was maintained. The pivotal study (D-02) $(N=235$ implanted, $N=222$ evaluable) consisted of two phases: a shortterm phase, and a second long-term phase of collecting data on safety and effectiveness of treatment (Rush et al. 2005a, 2005b). The short-term phase is a multicenter, doubleblind, randomised, controlled study with a parallel group not receiving treatment over 12 weeks (after implantation). All patients had an implanted with VNS. After two weeks, the device was activated in half of the patients. Patients were evaluated 10 weeks later. The two groups were then compared. Drug treatment was expected to remain stable throughout the short-term phase, both for the treatment group and for the control group. During the 2 weeks following the activation of VNS, the parameters were adjusted according to the tolerance of the subject, and remained stable during the remainder of the short-term phase. The 24-point Hamilton 
Depression Scale (HDRS-24) was the primary endpoint of the pivotal study (D-02). For the analysis of the short-term phase, the response rates on the HDRS-24 scale of the treatment and control group were compared. Secondary efficacy analyses included intraand inter-group comparisons with self-reported depressive symptom tests: IDS-SR, CGI, MADRS and MOS SF-36. The response was prospectively defined as a $\geq 50 \%$ increase in the IDS-SR, HDRS- 24 and MADRS scores compared to initial values, and as a significant or very significant improvement in CGI. Remission (full response) was prospectively defined as a score $\leq 9$ on the HDRS-24 scale, a score $\geq 10$ for the MADRS scale or a score $\leq 14$ for the IDS-SR test. After 10 weeks, the response was 15\% (active group) and 10\% (placebo group). This difference was not statistically different. The authors concluded that VNS has not demonstrated short-term efficacy in the treatment of depression. Reasons for the failure of the acute phase could have been: (1) an underpowering of the study; (2) a very resistant population; (3) a too-short time period for VNS therapy to demonstrate superiority; (4) underdosing of the active treatment arm. All subjects in the pivotal study (D-02) $(N=205$ subjects) at the end of the short-term phase were able to undertake the long-term extension phase, active treatment with the VNS system. The results showed a response rate of $27 \%$ and a remission rate of $15.8 \%$. The improvement in depression observed among subjects in the long-term phase of the pivotal study (D02) was corroborated by an improvement in the quality of life measured by the MOS SF-36 (Rush et al. 2005a, 2005b). This study was biased by the absence of a comparison group, the concomitant use of antidepressants, and the fact that treatments were adjusted during the study period for both the VNS and for antidepressants. The efficacy results of the D-03 study, which evaluated the efficacy and safety of VNS in 74 patients with TRD, showed that $53 \%$ of responders were responders (Schlaepfer et al. 2008). The results of this 2-year open-label study suggest a good clinical response and a relatively benign adverse event profile. However, the absence of a control group limits the validity of the results of this study. Long-term, comparative, observational, non-randomised long-term study D-04 represents a control group of subjects suffering from TRD and who have received usual drug treatments and for which the VNS device has not been implanted during the first 12 months (Bajbouj et al. 2010). During this time, a comparison was made between the two groups: 205 patients with VNS and usual treatment (TAU), which also included ECT, and 124 patients who received only TAU. The first group (VNS + TAU) showed a $27 \%$ improvement on the HDRS-24 scale, compared with the $13 \%$ improvement for the nonVNS group. Thus, primary and secondary analyses showed that adjuvant treatment with VNS resulted in statistically significant improvement in depressive symptoms after one year of treatment.

Most adverse effects of feasibility study D-01 and pivot study D-02 are minor or moderate. In study D-01, no interruption was associated with adverse events attributed to the VNS or implantation. In the pivotal study D-02, after one year, 3\% (8/235) of subjects discontinued treatment due to an adverse event. Adverse events considered to be related to implantation occurred in less than $5 \%$ of subjects during the short-term phase of pivot study D-02. Adverse events associated with stimulation had an incidence $\geq 5 \%$ in the pivotal study (D-02) and an incidence $<5 \%$ in the long-term phase of the same study. After the first 3 months of stimulation, the incidence of adverse effects related to stimulation did not exceed 1.3\%. Of the 295 subjects on which the device was implanted, both feasibility study D-01 and pivot study D- $02,92 \%$ of subjects continued treatment with the VNS system at 12 months and $82 \%$ at 24 months. Recent systematic reviews and meta-analyses of open-label studies have suggested that the antidepressant effects of VNS may accrue over time. By analysing participants in pivotal trials D-02 $(N=235)$, Nierenberg et al. showed that short-term and long-term results (24 months) for VNS treatment were similar for patients with unipolar depressive symptoms (33\%) and those with bipolar depression (38\%) (Nierenberg et al. 2008).

Daban et al. (2008) evaluated the safety and efficacy of VNS stimulation in TRD through a systematic review between January 2000 and September 2007. Of the 48 references found, only 18 studies $(N=1251)$ met the required quality criteria. Only one randomised double-blind study was available and therefore a meta-analysis was not possible. In the majority of preliminary open-label studies selected for examination, VNS was associated with a significant reduction in depressive symptoms (based on the primary endpoint, HDRS) in the short and long term. Unfortunately, the only double-blind study gave inconclusive results. In general, VNS is reported as a safe and feasible procedure, despite its invasiveness. The authors concluded that VNS appears to be an interesting new approach for the treatment of TRD. However, despite promising results reported primarily in open-label studies, further clinical trials are needed to confirm its efficacy in TRD. Martin and Martín-Sánchez (2012) conducted a systematic review and meta-analysis to determine the efficacy of VNS for the treatment of TRD. Fourteen studies met the inclusion criteria and were selected for analysis. The meta-analysis of the efficacy of uncontrolled studies showed a significant reduction in scores on the HDRS scale, and the percentage of responders was $31.8 \%$. However, as already mentioned, the randomised controlled trial $(R C T)$ for a sample of 235 patients with depression reported no statistically significant difference between the active and the placebo groups. To investigate the cause of this heterogeneity between studies, a meta-regression was performed. Eighty-four percent of this variation can be explained by the initial severity of depression. The authors indicated that the available data are insufficient to conclude whether or not the VNS is effective in the treatment of depression. Furthermore, it cannot be excluded that the positive results observed in the uncontrolled studies could have been mainly due to a placebo effect.

Berry et al. conducted a meta-analysis to compare response and remission rates in patients with TRD and treated with VNS in addition to the usual treatment (VNS + TAU), or TAU (Berry et al. 2013). The six clinical studies included in the meta-analysis were two single-arm studies of VNS + TAU, a randomised trial of VNS + TAU versus TAU, a single arm study of TAU patients, a randomised VNS + TAU comparing different intensities of VNS stimulation, and a non-randomised registry of patients who received either VNS + TAU or TAU. Evaluation of the response was measured on the MADRS and CGI-I scales. The results were compared with baseline with 96 weeks of treatment with VNS + TAU $(n=1035)$ versus TAU $(n=425)$. The response rate for VNS + TAU at $12,24,48$ and 96 weeks was $12 \%, 18 \%, 28 \%$ and $32 \%$, respectively, compared with $4 \%, 7 \%, 12 \%$ and $14 \%$, respectively, the TAU. The rate of remission on the MADRS for VNS + TAU at 12, 24, 48 and 96 weeks was $3 \%, 5 \%, 10 \%$ and $14 \%$ - against 1\%, $1 \%, 2 \%$ and $4 \%$ for TAU. The adjuvant VNS was associated with a higher probability of response and remission compared to TAU. Similar results were observed for the CGI-I response. The authors concluded that for patients with chronic TRD, VNS + TAU has response and remission rates that are more likely to persist over time in comparison with the TAU group. The most commonly reported adverse effects after 1 year of VNS for TRD are voice alteration $(69.3 \%)$, dyspnoea $(30.1 \%)$, pain $(28.4 \%)$, and increased 
Table 2. Depression: overview clinical pivotal studies.

\begin{tabular}{lc}
\hline D-01 (efficacy and safety) & D-03 (efficacy and safety) \\
Acute (3 months) & Acute and long-term (24 months) \\
D-02 (efficacy and safety) & D-21 (dosing) \\
Acute and long-term (24 months) & Acute and long-term (12 months) \\
D-02 vs. D-04 (efficacy and safety) VNS vs. VNS + TAU & D-23 (efficacy; safety; mortality; suicidality; anxious) TAU vs. TAU + VNS \\
Acute and long-term (12 months) & Long-term (60 months) \\
\hline
\end{tabular}

cough (26.4\%). Voice alteration and increased cough are often direct effects of VNS being actively delivered and can immediately improve by turning the stimulation off. A lower all-cause mortality rate, including suicide, has been observed in patients with TRD treated with adjunctive VNS compared to TAU (Olin et al. 2012). There is promising evidence for the use of VNS for depression in a 12-month (Aaronson et al. 2013; Christmas et al. 2013; Feldman et al. 2013; Müller et al. 2013).

Aaronson et al. (2013) in a multicenter, double-blind study compared the safety and efficacy of different levels of adjuvant therapy stimulation by VNS for TRD. The 331 patients were randomised to one of three dose groups: low $(0.25 \mathrm{~mA}, 130 \mu \mathrm{s}$ pulse width), mean $(0.5-1 \mathrm{~mA}, 250 \mu \mathrm{s})$ or high $(1.25-1.5 \mathrm{~mA}, 250 \mu \mathrm{s})$. A highly resistant population ( $>97 \%$ of subjects failed to respond to $\geq 6$ previous antidepressant treatments) was studied. Responses and side effects were evaluated for 22 weeks (end of acute phase), and thereafter the stimulation current could be increased if necessary. The evaluations then continued until week 50 (end of the long-term phase). During the acute phase, all groups showed statistically significant improvement on the primary efficacy endpoint (change in IDS-C score). The reported rates of serious adverse psychiatric events have included suicide or attempted suicide (4.6\%) and treatment-emergent hypomania or mania (2.7\%). In the longterm phase, the mean change in the IDS-C score showed continuous improvement. Post hoc analysis demonstrated a statistically significant correlation between the total daily load and the decrease in depressive symptoms; the acute phase analysis demonstrated significantly greater durability of response at medium and high doses than at low doses. The authors concluded that patients receiving adjuvant therapy with VNS showed a significant improvement at the end of the study compared to the initial values, the effect being lasting over 1 year. However, the absence of a control group is a bias for this study.

Smaller studies also showed high levels of remittance of TRD over longer periods ( $>5$ years). In the case control retrospective study published by Müller et al., a group of 20 patients with TRD was treated with low-strength/high-frequency VNS $(\leq 1.5 \mathrm{~mA}$, $20 \mathrm{~Hz})$ and highstrength/low-frequency $(>1.5 \mathrm{~mA}, 15 \mathrm{~Hz})$ VNS. Significant decrease in the HAMD was observed in patients who were treated with the low-strength/high-frequency stimulation parameters. The scores of the patients treated with high-strength/ low-frequency combination did not change. Sixty-percent of our patients had low-strength/high-frequency stimulation with frequency $30 \mathrm{~Hz}$ and $0.65 \pm 0.35 \mathrm{~mA}$ during the follow up period. The frequency was the first parameter to be increased when the patient demonstrated HAMD deterioration (Müller et al. 2017). In a recent study, the authors had compared the durations of response achieved with VNS + TAU vs. treatment as usual (TAU). VNS therapy added to TAU in severe TRD leads to rapid onset and higher likelihood and a greater durability of the response as compared to TAU alone. In the VNS + TAU arm, $62.5 \%$ of participants had a first response over 5 years compared with $39.9 \%$ in TAU. The time to first response was significantly shorter for VNS + TAU than for TAU. This is the first study to look at the long-term durability of benefit up to 5 years, defined here as response based on $50 \%$ reduction in baseline MADRS score to declare the onset of response and a threshold of retaining at least a $40 \%$ reduction compared to baseline to retain the response (Kumar et al. 2019).

The TRD registry investigated whether adjunctive VNS with TAU in depression has superior long-term outcomes compared with TAU only: a 5 year, prospective, open-label, nonrandomised, observational registry study was conducted at 61 U.S. sites and included 795 patients with TRD (Aaronson et al. 2017). This registry represents the longest and largest naturalistic study of efficacy outcomes in TRD. The adjunctive VNS group had better clinical outcomes than the treatment-as-usual group, including a significantly higher 5-year cumulative response rate $(67.6 \%$ compared with $40.9 \%$ ) and a significantly higher remission rate (cumulative first-time remitters, $43.3 \%$ compared with $25.7 \%$ ).

Recently, Aaronson et al. provided a large set of data showing improved outcomes for adjunctive VNS observed in both ECT responders and non-responders. Within the D-23 VNS registry (489 in the VNS arm and 276 in the treatment-as-usual arm), cumulative remission, based on an MADRS total score, demonstrated that over time, patients in the VNS arm were significantly more likely to experience remission than those in the treatment-as-usual arm (43.3 and $25.7 \%$, respectively), demonstrating significant efficacy. The 5-year cumulative response rate for patients in the VNS arm who had previously responded to ECT was $71.3 \%$ compared with $56.9 \%$ for the ECT responders in the treatment-as usual arm. A similar significant response differential was observed among ECT nonresponders (59.6\% compared with 34.1\%) (Aaronson et al. 2017).

\section{Discussion}

We have previously observed that in the part dedicated to the description of the trials performed with the VNS under the TRD, all studies examined (D-01, D02, D03, D04, D21 and D23) were for the most part observational and without placebo control (Table 2).

In a recent study, Bottomley et al. provided an up-to-date systematic literature reviews (SLRs) and meta-analysis of all studies of adjunctive VNS in TRD, including recent much longer-term experience of VNS (Bottomley et al. 2020). Of 22 identified studies, there were two RCT (Rush et al. 2005a, 2005b; Aaronson et al. 2013), 16 single-arm and four non-randomised comparative studies. The only RCT $(N=235)$ reported no statistically significant differences between the active and placebo groups (Rush et al. 2005a, 2005b). This study revealed modest benefit per the primary measure. Response rates with a secondary outcome, the IDSSR, were noted to demonstrate a statistically significant benefit for VNS. The authors considered that the lack of definitive evidence of shortterm efficacy in this chronically ill population could be due to either the study being too short in its follow up or underpowered.

There are authors who consider that the effectiveness of VNS therapy for TRD remains modest. For example, in a meta-analysis, Martin and Martín-Sánchez (2012) stated that there is insufficient evidence to conclude that VNS is effective in treating TRD. They suggest that its positive effects could be influenced by the placebo effect, spontaneous remission, or simply by observing these patients (Hawthorne's effect). However, in their analysis, these authors mix epilepsy studies and depression studies. Strong evidence of a possible antidepressant effect should be based 
Table 3. D-23 VNS registry.

VNS therapy superior to TAU over 5-year period

VNS therapy patients experienced statistically significant benefits compared to

TAU patients for $13 / 15$ clinical outcomes and 2 of 4 quality of life/ health outcomes

Physician-rated and self-rated symptomatology response rates, times to response, duration of response, and quality of life consistently favoured the VNS therapy compared to TAU

exclusively on long-term clinical trials with a control group, but in reality, this objective appears to be problematic, as there are significant barriers, both, technical and ethical. It is important to highlight the special nature of the population of patients enrolled in the VSN therapy studies, which is distinguished by the chronicity of their depressive disorder (the average length of the disease may be more than 25 years, and of the current episode nearly 7 years). It is important to note that there is a real drug resistance, with an important therapeutic escape $(>50 \%$ of patients who have failed on average more than 7 antidepressant treatments and ECT). It was also necessary to count the total number of hospitalisations, and especially the number of attempted suicides. These patients have always been excluded from most clinical trials with antidepressant treatments (except for DBS studies). If we take into account all of these clinical features, it will be difficult to say that a placebo effect, spontaneous remission or Hawthorne's effect may explain even modest improvements in these patients. As with conventional antidepressant treatments, the efficacy of VNS on depression appears to be proportional to the severity of the initial chart, thus explaining the variations in efficacy observed throughout the studies. The second RCT (Aaronson et al. 2013) compared three active adjunctive VNS treatment groups. The patients were randomised to three different levels of VNS dose stimulation. All three groups showed statistically significant improvement in the primary endpoint. A post hoc analysis suggested higher doses may result in better and more sustained responses.

Since its introduction in 2000, numerous studies have been published and SLRs. Seven SLRs have reported that adjunctive VNS therapy (namely VNS therapy plus TAU is associated with patient benefit in TRD, and that such benefit is sustained (Bottomley et al. 2020). There are the similarities and differences across the seven SLRs:

a. Three focussed solely on TRD (Daban et al. 2008; Berry et al. 2013; Lv et al. 2019). Lv et al. searched for RCTs in TRD, and identified two RCTs: one was VNS therapy (Rush et al. 2005a, 2005b); the second study described auricular transcutaneous VNS as opposed to VNS therapy.

b. Two SLRs included other conditions: TRD/drug-resistant epilepsy (Martin and Martín-Sánchez 2012) and experiences in any psychiatric condition (Cimpianu et al. 2017);

c. One SLR (Milev et al. 2016) was a clinical guideline in adults with TRD based on the meta-analyses of therapeutic neuromodulation (McGirr and Berlim 2018).

This comprehensive study of all VNS data, offers a summary of the best available evidence, suggesting that adjunctive VNS over a 2-year period, make a true benefit for TRD patients.

The neuromodulation of VNS gained a special interest in recent years. In order to improve the response to antidepressant treatment, add-on augmentation has been developed. In addition to pharmacological add-on therapy, neurostimulation techniques are increasingly used. Concerning the combining of VNS with existing treatments, VNS is a promising add-on treatment for TRD (Cimpianu et al. 2017). One of the distinct advantages of VNS is that it can be combined with safely with a wide range of medications and with all existing treatments for depression.

Aaronson et al. published the report of the longest and largest treatment trial of VNS use in TRD. The investigators suggested that patients with a history of positive ECT response did especially well over time with VNS + TAU. Thys study demonstrated that VNS combined with any treatment available to psychiatrists (including ECT) was superior to standard treatment without VNS, achieving greater cumulative response $(50 \%$ reduction in depressive symptoms) and remission rates and lowering overall suicide rates. These results show that VNS is a feasible adjunctive tool for ECT responders. These two neuromodulation modalities of treatment should be viewed as complementary. The VNS device can be temporarily shut off to permit ECT to be administered and then restarted immediately post ECT. VNS has many scientific evidence for efficacy. One important point in response-rates of VNS is the latency between implantation and clinical response, so, its therapeutic benefit is clearly slower than ECT. On the contrary, its efficacy increase with time and the longer-term results of VNS are encouraging. VNS conferring the advantage of a very well tolerated longterm treatment. For maintenance in long term treatment, it is better even than the maintenance ECT, and also less invasive (Müller et al. 2018). Both devices provide additional options for the large number of patients. In recent years, a growing interest in cognitive dysfunction in depression has emerged. VNS seems a very promising adjunctive therapy for TRD patients with cognitive impairment. Unlike ECT, it does not cause adverse cognitive effects. Desbeaumes et al. suggested that chronic VNS produces sustained clinical and cognitive improvements in TRD patients, with some mental functions improving as soon as 1 month after the initiation of the VNS therapy (Desbeaumes et al. 2018).

Results from a non-randomised registry of VNS + TAU vs. TAU $(n=636)$ suggest that adjunctive VNS demonstrated significant advantages in the mood domain, and in multiple other functional domains measured on the Q-LES-Q-SF, with significant comparative benefit evident from 3 months and sustained through 5 years on VNS + TAU (Conway et al. 2018) (Table 3). A long-term (a 6year follow-up) naturalistic study demonstrated that the therapeutic effect of VNS on TRD goes beyond clinical symptoms to improve the daily quality of life of those affected (Trottier-Duclos et al. 2018). The benefit of VNS for TRD accrues over time, based upon prospective observational studies that followed patients for up to one or more years. An ongoing clinical trial, a study to assess effectiveness and efficiency of VNS therapy in patients with difficult to treat depression (RESTORE-LIFE) is in progress. The primary objective of this study is to assess short, mid and long-term clinical outcomes in patients with TRD treated with VNS as adjunctive therapy (a minimum of 500 patients and up to 80 sites, 5 years) (ClinicalTrials.gov database).

The tolerance and safety of the VNS systems has been well established (Cristancho et al. 2011; Ben-Menachem et al. 2015). This was reflected in patient's adherence to treatment: in the pivotal study, the continuation rate of one-year VNS therapy was high $-90 \%$, and at two years more than $80 \%$ of patients have the device in place. We have seen that in all studies, hypomanic symptoms were very rare, short-lived and responded to a temporary reduction in the intensity of VNS. In trials conducted to date, it appears that VNS therapy does not appear to exacerbate depressive symptoms or specifically induce suicidal tendencies. 


\section{Conclusions}

After studying the literature, we conclude that VNS therapy for TRD has two distinct features that differentiate it from other antidepressant treatments: as sustained efficacy (response and remission) obtained in highly resistant depressive disorders, and a favourable safety profile. This therapy has no negative effects on cognition. In general, studies often have limitations such as lack of control group, small sample size, concomitant drug therapy, lack of statistical power analysis, and short-term follow-up. The VNS still requires further studies on optimal stimulation parameters (Yuan and Silberstein 2016). Despite its advantages, VNS remains an invasive technique and it requires multidisciplinary teams involving psychiatrists and neurosurgeons. VNS is recommended as a thirdline acute treatment with level 3 evidence for efficacy (Milev et al. 2016). However, the level of evidence as chronic treatment is 2 . The analysis of the results of VNS for TRD and especially the comparison between the different techniques of neuromodulation is difficult, sometimes impossible. Most patients with VNS are on antidepressant medications, so adverse effects are for the combined treatment. VNS appears to be a very safe form of treatment. In addition to the antidepressive mode of action, a remarkable finding is that VNS seems to have a specific lower all-cause mortality rate and an anti-suicidal effect (Aaronson et al. 2013, 2017; Berry et al. 2013). The tolerability of VNS appears to improve over time with diminishing rates of adverse events reported by patients during their long-term treatment with VNS. Of course, the sustained efficacy is a very important element and the results with VNS appear encouraging (Ben-Menachem et al. 2015).

VNS exhibits antidepressive and augmentative effects and this therapy appears to be an important new addition to the therapeutic arsenal of the clinician treating patients with TRD. The augmentation effects of combining neurostimulation techniques (alternative and/or add-on strategies) are very interesting (e.g., ECT followed by VNS). There are promising results if we apply this new strategy and it should be strongly recommended (Müller et al. 2018). In situations where treatment adherence may be an issue, VNS can be considered a good therapeutic choice for patients with chronic depression. The standard guidelines contribute to facilitate and guide the treatment decision and choice for the clinicians. However, they cannot take into account the overall complexities involved in the care of each individual patient.

\section{Acknowledgements}

The authors are responsible for the writing and the content of the paper.

\section{Disclosure statement}

No potential conflict of interest was reported by the author(s).

\section{References}

Aalbers M, Vles J, Klinkenberg S, Hoogland G, Majoie M, Rijkers K. 2011. Animal models for vagus nerve stimulation in epilepsy. Exp Neurol. 230(2):167-175.

Aaronson ST, Carpenter LL, Conway CR, Reimherr FW, Lisanby SH, Schwartz TL, Moreno FA, Dunner DL, Lesem MD, Thompson PM, et al. 2013. Vagus nerve stimulation therapy randomized to different amounts of electrical charge for treatment-resistant depression: acute and chronic effects. Brain Stimul. 6(4):631-640.

Aaronson ST, Sears P, Ruvuna F, Bunker M, Conway CR, Dougherty DD, Reimherr FW, Schwartz TL, Zajecka JM. 2017. A 5-year observational study of patients with treatment-resistant depression treated with vagus nerve stimulation or treatment as usual: comparison of response, remission, and suicidality. Am J Psychiatry. 174(7):640-648.

Bajbouj M, Merkl A, Schlaepfer TE, Frick C, Zobel A, Maier W, O'Keane V, Corcoran C, Adolfsson R, Trimble M, et al. 2010. Two-year outcome of vagus nerve stimulation in treatmentresistant depression. J Clin Psychopharmacol. 30:273-281.

Ben-Menachem E, Revesz D, Simon BJ, Silberstein S. 2015. Surgically implanted and non-invasive vagus nerve stimulation: a review of efficacy, safety and tolerability. Eur J Neurol. 22(9): 1260-1268.

Berry SM, Broglio K, Bunker M, Jayewardene A, Olin B, Rush AJ. 2013. A patient-level metaanalysis of studies evaluating vagus nerve stimulation therapy for treatment-resistant depression. Med Devices. 6:17-35.

Bottomley JM, Lereun C, Diamantopoulos A, Mitchell S, Gaynes BN. 2020. Vagus nerve stimulation (VNS) therapy in patients with treatment resistant depression: a systematic review and meta-analysis. Comprehensive Psychiatry. 98:152156.

Chae J-H, Nahas Z, Lomarev M, Denslow S, Lorberbaum JP, Bohning DE, George MS. 2003. A review of functional neuroimaging studies of vagus nerve stimulation (VNS). J Psychiatr Res. 37(6):443-455.

Christmas D, Steele JD, Tolomeo S, Eljamel MS, Matthews K. 2013. Vagus nerve stimulation for chronic major depressive disorder: 12-month outcomes in highly treatment-refractory patients. J Affect Disord. 150(3):1221-1225.

Cimpianu C-L, Strube W, Falkai P, Palm U, Hasan A. 2017. Vagus nerve stimulation in psychiatry: a systematic review of the available evidence. J Neural Transm (Vienna). 124(1):145-158.

Conway CR, Chibnall JT, Gebara MA, Price JL, Snyder AZ, Mintun MA, Craig ADB, Cornell ME, Perantie DC, Giuffra LA, et al. 2013. Association of cerebral metabolic activity changes with vagus nerve stimulation antidepressant response in treatment-resistant depression. Brain Stimul. 6(5):788-797.

Conway CR, Kumar A, Xiong W, Bunker M, Aaronson ST, Rush AJ. 2018. Chronic vagus nerve stimulation significantly improves quality of life in treatment-resistant major depression. J Clin Psychiatry. 79(5):18m12178.

Conway CR, Xiong W. 2018. The mechanism of action of vagus nerve stimulation in treatmentresistant depression: current conceptualizations. Psychiatr Clin North Am. 41(3):395-407.

Cristancho P, Cristancho MA, Baltuch GH, Thase ME, O' Reardon JP. 2011. Effectiveness and safety of vagus nerve stimulation for severe treatment-resistant major depression in clinical practice after FDA approval: outcomes at 1 year. J Clin Psychiatry. 72(10):1376-1382.

Cyberonics. 2015. VNS therapy system physician's manual. Houston (TX): Cyberonics Inc.

Daban C, Martinez-Aran A, Cruz N, Vieta E. 2008. Safety and efficacy of vagus nerve stimulation in treatment-resistant depression: a systematic review. J Affect Disord. 110(1-2):1-15.

Desbeaumes JV, Richer F, Miron JP, Fournier-Gosselin MP, Lespérance P. 2018. Long-term sustained cognitive benefits of vagus nerve stimulation in refractory depression. J ECT. 34:283-290.

Feldman RL, Dunner DL, Muller JS, Stone DA. 2013. Medicare patient experience with vagus nerve stimulation for treatmentresistant depression. J Med Econ. 16(1):62-74.

Follesa P, Biggio F, Gorini G, Caria S, Talani G, Dazzi L, Puligheddu $M$, Marrosu F, Biggio G. 2007. Vagus nerve stimulation increases norepinephrine concentration and the gene expression of BDNF and bFGF in the rat brain. Brain Res. 1179:28-34. 
Furmaga H, Carreno FR, Frazer A. 2012. Vagal nerve stimulation rapidly activates brain-derived neurotrophic factor receptor TrkB in rat brain. PLoS One. 7(5):e34844.

Grimonprez A, Raedt R, Baeken C, Boon P, Vonck K. 2015. The antidepressant mechanism of action of vagus nerve stimulation: evidence from preclinical studies. Neurosci Biobehav Rev. 56: 26-34.

Groves DA, Brown VJ. 2005. Vagal nerve stimulation: a review of its applications and potential mechanisms that mediate its clinical effects. Neurosci Biobehav Rev. 29(3):493-500.

Hulsey DR, Shedd CM, Sarker SF, Kilgard MP, Hays SA. 2019. Norepinephrine and serotonin are required for vagus nerve stimulation directed cortical plasticity. Exp Neurol. 320:112975.

Kumar A, Bunker MT, Aaronson ST, Conway CR, Rothschild AJ, Mordenti G, Rush AJ. 2019. Durability of symptomatic responses obtained with adjunctive vagus nerve stimulation in treatmentresistant depression. Neuropsychiatr Dis Treat. 15:457-468.

Liu W, Ge T, Leng Y, Pan Z, Fan J, Yang W, Cui R. 2017. The role of neural plasticity in depression: from hippocampus to prefrontal cortex. Neural Plast. 2017:11.

Lv H, Zhao Y-H, Chen G-J, Wang D-Y, Chen H. 2019. Vagus nerve stimulation for depression: a systematic review. Front Psychol. 10:64.

Martin JLR, Martín-Sánchez E. 2012. Systematic review and metaanalysis of vagus nerve stimulation in the treatment of depression: variable results based on study designs. Eur Psychiatr. 27(3):147-155.

McGirr A, Berlim MT. 2018. Clinical usefulness of therapeutic neuromodulation for major depression: a systematic meta-review of recent meta-analyses. Psychiatr Clin North Am. 41(3):485-503.

Mehta V, Kramer D, Russin J, Liu C, Amar A. 2018. Chapter 82: vagal nerve simulation. In: Elliot Krames, Ali Rezai, and Hunter Peckham, editors. Neuromodulation. Comprehensive Textbook of Principles, Technologies, and Therapies. Book.2nd ed. p. 999-1009.

Milev RV, Giacobbe P, Kennedy SH, Blumberger DM, Daskalakis ZJ, Downar J, Modirrousta M, Patry S, Vila-Rodriguez F, Lam RW, et al. 2016. Clinical guidelines for the management of adults with major depressive disorder: section 4. Neurostimulation treatments. Can J Psychiatry. 61(9):561-575.

Müller HH, Kornhuber J, Maler JM, Sperling W. 2013. The effects of stimulation parameters on clinical outcomes in patients with vagus nerve stimulation implants with major depression. J ECT. 29:e40-e42.

Müller HH, Lücke C, Moeller S, Philipsen A, Sperling W. 2017. Efficacy and long-term tuning parameters of vagus nerve stimulation in long-term treated depressive patients. J Clin Neurosci. 44:340-341.

Müller HH, Moeller S, Lam A, Lücke C, Braun N, Philipsen A. 2018. Vagus nerve stimulation (VNS) and other augmentation strategies for therapy-resistant depression (TRD): review of the evidence and clinical advice for use. Front Neurosci. 12:239.

Nahas Z, Marangell LB, Husain MM, Rush AJ, Sackeim HA, Lisanby SH, Martinez JM, George MS. 2005. Two-year outcome of vagus nerve stimulation (VNS) for treatment of major depressive episodes. J Clin Psychiatry. 66(9):1097-1104.

Nahas Z, Teneback C, Chae J-H, Mu Q, Molnar C, Kozel FA, Walker J, Anderson B, Koola J, Kose S, et al. 2007. Serial vagus nerve stimulation functional $\mathrm{MRI}$ in treatment-resistant depression. Neuropsychopharmacology. 32(8):1649-1660.

Nemeroff CB, Mayberg HS, Krahl SE, McNamara J, Frazer A, Henry TR, George MS, Charney DS, Brannan SK. 2006. VNS therapy in treatment-resistant depression: clinical evidence and putative neurobiological mechanisms. Neuropsychopharmacology. 31(7): 1345-1355.

Nierenberg AA, Alpert JE, Gardner-Schuster EE, Seay S, Mischoulon D. 2008. Vagus nerve stimulation: 2-year outcomes for bipolar versus unipolar treatment-resistant depression. Biol Psychiatry. 64(6):455-460.

Olin B, Jayewardene AK, Bunker M, Moreno F. 2012. Mortality and suicide risk in treatment-resistant depression: an observational study of the long-term impact of intervention. PLoS One. 7(10): e48002.

Penry JK, Dean JC. 1990. Prevention of intractable partial seizures by intermittent vagal stimulation in humans: preliminary results. Epilepsia. 31(s2):S40-S43.

Perini Gl, Toffanin T, Pigato G, Ferri G, Follador H, Zonta F, Pastorelli C, Piazzon G, Denaro L, Rolma G, et al. 2017. Hippocampal gray volumes increase in treatment-resistant depression responding to vagus nerve stimulation. J ECT. 33: 160166.

Revesz D, Tjernstrom M, Ben-Menachem E, Thorlin T. 2008. Effects of vagus nerve stimulation on rat hippocampal progenitor proliferation. Exp Neurol. 214(2):259-265.

Ruffoli R, Giorgi FS, Pizzanelli C, Murri L, Paparelli A, Fornai F. 2011. The chemical neuroanatomy of vagus nerve stimulation. J Chem Neuroanat. 42(4):288-296.

Rush AJ, George MS, Sackeim HA, Marangell LB, Husain MM, Giller C, Nahas Z, Haines S, Simpson RK, Goodman R. 2000. Vagus nerve stimulation (VNS) for treatment-resistant depressions: a multicenter study. Biol Psychiatry. 47(4):276-286.

Rush AJ, Marangell LB, Sackeim HA, George MS, Brannan SK, Davis SM, Howland R, Kling MA, Rittberg BR, Burke WJ, et al. 2005a. Vagus nerve stimulation for treatment-resistant depression: a randomized, controlled acute phase trial. Biol Psychiatry. 58(5): 347-354.

Rush AJ, Sackeim HA, Marangell LB, George MS, Brannan SK, Davis SM, Lavori P, Howland R, Kling MA, Rittberg B, et al. 2005b. Effects of 12 months of vagus nerve stimulation in treatmentresistant depression: a naturalistic study. Biol Psychiatry. 58(5): 355-363.

Rush AJ, Siefert SE. 2009. Clinical issues in considering vagus nerve stimulation for treatment-resistant depression. Exp Neurol. 219(1):36-43.

Sackeim HA, Keilp JG, Rush AJ, George MS, Marangell LB, Dormer JS, Burt T, Lisanby SH, Husain M, Cullum CM, et al. 2001. The effects of vagus nerve stimulation on cognitive performance in patients with treatment-resistant depression. Neuropsychiatry Neuropsychol Behav Neurol. 14(1):53-62.

Schlaepfer TE, Frick C, Zobel A, Maier W, Heuser I, Bajbouj M, O'Keane V, Corcoran C, Adolfsson R, Trimble M, et al. 2008. Vagus nerve stimulation for depression: efficacy and safety in a European study. Psychol Med. 38(5):651-661.

Trottier-Duclos F, Desbeaumes Jodoin V, Fournier-Gosselin M-P, Richer F, Desjardins N, Tieu S, Lespérance P. 2018. A 6-year follow-up study of vagus nerve stimulation effect on quality of life in treatmentresistant depression: a pilot study. J ECT. 34(4): E58-E60.

Vonck KJ, Larsen LE. 2018. Chapter 18. Vagus nerve stimulation: mechanism of action. In: Krames E, Peckham PH, Rezai AR, editors. Neuromodulation: comprehensive textbook of principles, technologies, and therapies. Vol. 1. London, United Kingdom: Elsevier. p. 211-220.

Yuan H, Silberstein S. 2016. Vagus nerve and vagus nerve stimulation, a comprehensive review: part II. Headache. 56(2):259-266. 